\title{
Utilización de macroinvertebrados acuáticos como herramienta para determinar la calidad del agua en la quebrada Santo Tomás, municipio de Pensilvania, Colombia
}

\author{
○ Sergio Adrián Murillo-Montoya $a^{1,2, *}$, $\odot$ Alexander Mendoza-Mora ${ }^{1,2}$, \\ $\odot$ Edwin Santiago Restrepo-Bastidas ${ }^{1,2}$, $\odot$ Miguel Ángel Rodríguez ${ }^{2,3}$
${ }^{1}$ Grupo de Investigación en Recursos Naturales, Biotecnología y Bioprospección (RENABBIO), Sistema de investigación, desarrollo tecnológico e innovación (SENNOVA), Centro Pecuario y Agroempresarial, La Dorada, Colombia
${ }^{2}$ Semillero de Investigación IDEAS. Servicio Nacional de Aprendizaje (SENA), Regional Caldas-Sede Puerto Boyacá, Colombia
${ }^{3}$ Estudiante-Aprendiz, Servicio Nacional de Aprendizaje, SENA, La Dorada, Colombia

\begin{abstract}
Resumen
A pesar de la riqueza hídrica del municipio de Pensilvania, Caldas, y del creciente uso de los macroinvertebrados acuáticos como herramienta sencilla, eficiente y de bajo costo para determinar la calidad del agua en diversos ecosistemas, no se conocen estudios de este tipo en el municipio. El objetivo de esta investigación fue evaluar la diversidad de los macroinvertebrados acuáticos y la calidad del agua de la quebrada Santo Tomás en el municipio de Pensilvania. Se recolectaron muestras de macroinvertebrados entre junio y agosto de 2015 en diferentes hábitats (hojarasca sumergida, sedimento fino y rocas), empleando una red Surber. Se registraron 2.175 individuos pertenecientes a 97 grupos taxonómicos; los órdenes más abundantes fueron Trichoptera y Ephemeroptera (54 \%). No se encontraron diferencias significativas $(p>0,05)$ en términos de diversidad entre los sitios evaluados y según los índices BMWP/Col y EPT, las aguas son muy limpias, aunque el índice ASPT indicó la presencia de aguas ligera y moderadamente contaminadas. (C) 2018. Acad. Colomb. Cienc. Ex. Fis. Nat.

Palabras clave: Diversidad biológica; Bioindicador; Fuente hídrica; Acueductos rurales; Monitoreo; Ecosistemas acuáticos.

Utilization of aquatic macroinvertebrates as a tool to determine water quality in Santo Tomás ravine, Pensilvania municipality (Colombia)

Abstract

Despite the water wealth of the municipality of Pensilvania, Caldas, and the growing use of aquatic macroinvertebrates as a simple, efficient and low-cost tool to determine water quality in various ecosystems, no studies of this type are known in the municipality. The objective of this research was to evaluate the diversity of aquatic macroinvertebrates and the water quality of the Santo Tomás stream in the municipality of Pennsylvania. Samples of macroinvertebrates were collected between June and August 2015 in different habitats (submerged leaf litter, fine sediment, and rocks), using a Surber net. There were 2,175 individuals belonging to 97 taxonomic groups; the most abundant orders were Trichoptera and Ephemeroptera (54\%). No significant differences were found ( $p>0.05)$ in terms of diversity between the evaluated sites and, according to the BMWP / Col and EPT indices, the waters are very clean, although the ASPT index indicated the presence of lightly to moderately polluted waters. (C) 2018. Acad. Colomb. Cienc. Ex. Fis. Nat.
\end{abstract}

Key words: Biological diversity; Bioindicadors; Water sources; Rural aqueducts; Monitoring; Aquatic ecosystems.

\section{Introducción}

Los ecosistemas acuáticos se destacan por los bienes y servicios ecosistémicos y, en términos de su valor biológico, por la gran diversidad de especies que pueden albergar (Jonsson, et al., 2001). Sin embargo, la demanda de este recurso y las actividades antrópicas generan modificaciones que afectan la biota residente, lo que constituye una creciente amenaza para los ecosistemas y la calidad del recurso hídrico (Dudgeon, et al., 2006).
Actividades cotidianas como el vertimiento de aguas residuales y la pérdida de vegetación ribereña para expandir la frontera agrícola y ganadera, son aspectos que contribuyen a acelerar la pérdida de la biodiversidad (Jorcin \& Nogueira, 2008; Giraldo, et al., 2014), ya que modifican la

*Correspondencia:

Sergio Adrián Murillo-Montoya, samurillo@sena.edu.co

Recibido: 2 de mayo de 2018

Aceptado: 6 de julio de 2018

Editor: Gabriel Roldán 
estructura y el funcionamiento de las quebradas provocando la alteración de los regímenes de flujo de agua, el aumento de la carga de sedimentos y de nutrientes potencialmente contaminantes, así como variaciones en los niveles de luz, lo cual homogeneiza la biodiversidad (Delong \& Brusven, 1998; Roldán, 1999; Sweeney, et al., 2004).

En los ecosistemas acuáticos los macroinvertebrados se han utilizado ampliamente como herramienta para determinar la calidad del agua (Roldán, 1999; Zúñiga, 2010; Fierro, et al., 2015) debido, entre otras razones, a que su taxonomía es relativamente sencilla, son abundantes y diversos, los materiales y métodos de muestreo están estandarizados y son de bajo costo y, además, responden a agentes contaminantes, lo que permite diagnosticar la calidad y el estado de conservación del cuerpo de agua en el que habitan (Roldán, 1999).

Los estudios de este tipo son escasos en el departamento de Caldas, siendo representativos los trabajos de HanhVon Hessberg, et al. (2009) en la estación piscícola de la granja Montelindo (municipio de Palestina), de WalterosRodríguez \& Paiba-Alzate (2010) en las quebradas El Diamante, La Cristalina y Rio Chinchina de la reserva Torre Cuatro (Manizales), de González, et al. (2012), de Mesa-S, et al. (2013) en la cuenca alta del río Chinchiná (municipio de Manizales), y de Gutiérrez \& Días (2015), quienes evaluaron la diversidad de Ephemeroptera en 12 municipios del departamento. La mayoría de estos trabajos se han realizado en la zona centro sur, en tanto que la zona oriente se ha estudiado poco, aunque se destacan las investigaciones de Walteros-Rodríguez \& Daza-Castro (2009) en el río Guarinó (municipio de Manzanares) y de García, et al. (2013) en el río Manzo (municipio de Norcasia).

Dada la falta de información sobre este tema en el oriente del departamento y su desconocimiento en el municipio de Pensilvania, el presente estudio tuvo como objetivo evaluar la diversidad de macroinvertebrados acuáticos y la calidad del agua de la quebrada Santo Tomás (Pensilvania), como línea de referencia para programas de monitorización y conservación de las fuentes hídricas que abastecen los acueductos rurales, y con el fin de implementar a corto plazo estrategias de biomonitorización participativa.

\section{Materiales y métodos}

Area de estudio. El estudio se hizo en la quebrada Santo Tomás, en el corregimiento de Arboleda-Pensilvania, al oriente del departamento de Caldas (Figura 1). La cuenca hace parte de la hoya hidrográfica del río Samaná Sur, tiene una temperatura media de $22{ }^{\circ} \mathrm{C}$ y la pluviosidad alcanza los $3.500 \mathrm{~mm}$ anuales, con pendientes superiores al $50 \%$ (Corpocaldas, 1999).

Se realizaron tres muestreos durante los meses de junio, julio y agosto de 2015, uno por mes, correspondiente a la transición entre el periodo seco y el inicio de las lluvias, con una pluviosidad promedio de $111 \mathrm{~mm}$ en junio, $101 \mathrm{~mm}$ en julio y 85 mm en agosto (Agroclima, 2015).

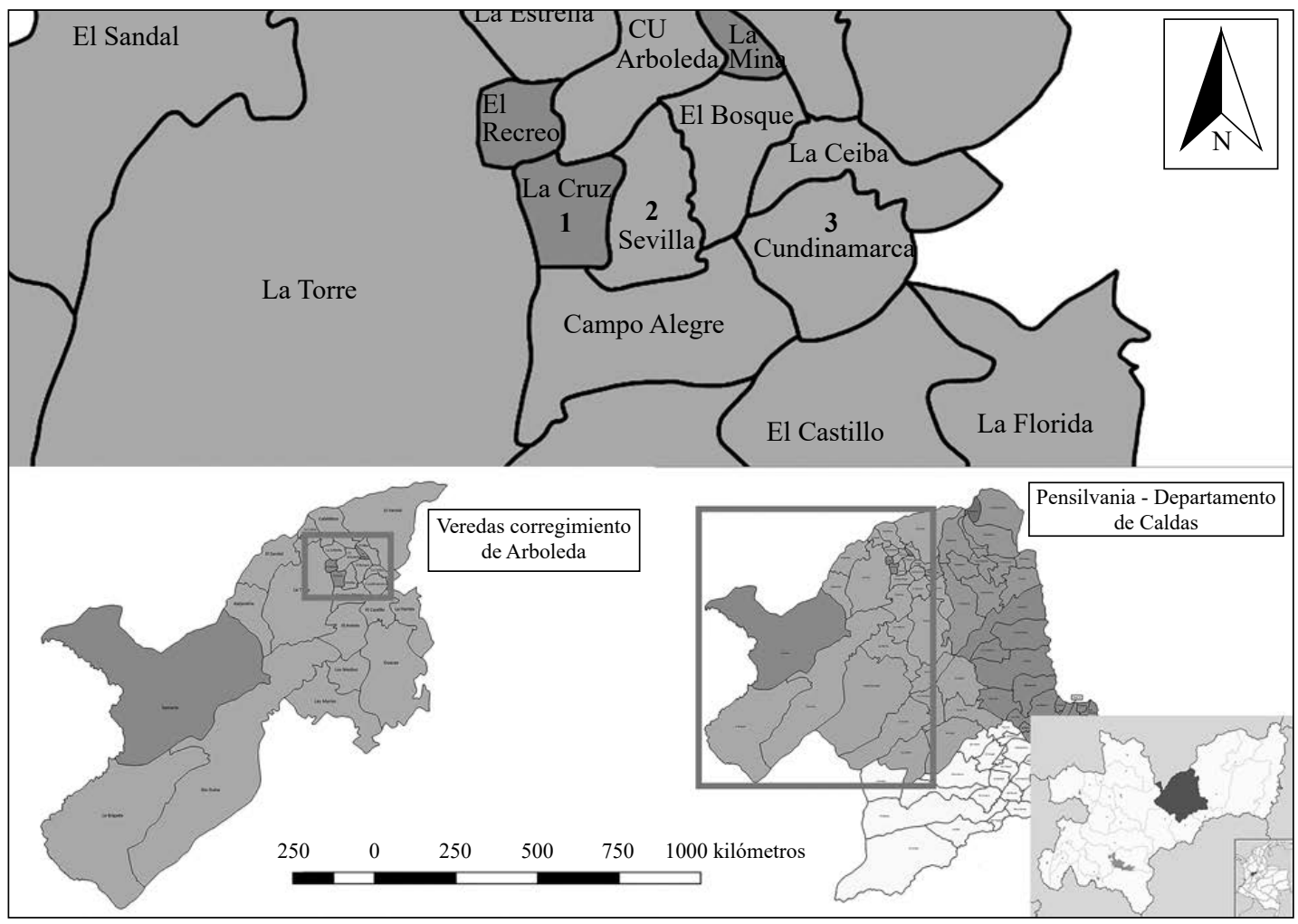

Figura 1. Quebrada Santo Tomás, Arboleda-Pensilvania, Caldas. Los números indican los sitios de muestreo: 1: vereda La Cruz, 2: vereda Sevilla y 3: vereda Cundinamarca 
En la quebrada se seleccionaron tres sitios de muestreo. El primero en la vereda La Cruz, a $1.570 \mathrm{msnm}$ en las coordenadas 5'29'29" N 7501'11,1'O. Este sitio hace parte de un área conservada por la junta del acueducto local desde 1999; la vegetación ribereña esta dominada por árboles y arbustos de las familias Rubiaceae, Melastomatacea, Lauraceae y Piperacea; el ancho del cauce es de $1,75 \mathrm{~m}$, el caudal promedio, de $126 \mathrm{l} / \mathrm{s}$ y la profundidad de $0,45 \mathrm{~m}$. El segundo punto de muestreo se ubicó en la vereda Sevilla, situada a $1.311 \mathrm{msnm}$ en las coordenadas $5^{\circ} 29^{\prime} 56,3^{\prime \prime} \mathrm{N}$ y $75^{\circ} 10^{\prime} 42,72^{\prime}$ 'O. Este sitio recibe descargas de aguas residuales del corregimiento de Arboleda y la vegetación ribereña asociada es de tipo de las herbáceas, con una prevalencia de piperaceas y melastomataceas; el ancho del cauce es de $2,5 \mathrm{~m}$, el caudal promedio, de $238 \mathrm{l} / \mathrm{s}$ y la profundidad de $0,96 \mathrm{~m}$. El tercer punto se ubicó en la vereda Cundinamarca, a $1.270 \mathrm{msnm}$ en las coordenadas $5^{\circ} 28^{\prime} 49^{\prime \prime} \mathrm{N}$ y $75^{\circ} 06^{\prime} 18,7^{\prime \prime} \mathrm{O}$. En este sitio la vegetación ribereña es escasa, el uso del suelo es de vocación agrícola y ganadera; el ancho del cauce es de 7,6 m, el caudal promedio de 496 1/s y la profundidad de 1,3 m. Los sitios se seleccionaron también por su facilidad de acceso.

Recolección de macroinvertebrados y determinación taxonómica. En cada sitio de estudio se recolectaron los macroinvertebrados empleando una red Surber con un área de $0,045 \mathrm{~m}^{2}$ y un ojo de malla de $250 \mu \mathrm{m}$. Este mismo esfuerzo de muestreo se hizo en cada sustrato (hojarasca retenida, sedimiento fino y rocas) empleando un minuto por sustrato y cinco repeticiones (Ramírez, 2010). Las muestras se separaron con pinzas entomológicas y se conservaron en alcohol al $96 \%$; la identificación taxonómica se hizo en el laboratorio de ecología de la Universidad de Caldas y en el laboratorio de microbiología y análisis fisicoquímicos de aguas y alimentos del SENA, subregional La DoradaCaldas. Se emplearon las claves taxonómicas de Posada \& Roldán (2003), Manzo (2005; 2013), Heckman (2006; 2011), Domínguez \& Fernández (2009), Springer (2010), Gutiérrez \& Días (2015) para identificar el material recolectado, el cual fue depositado en la Colección Entomológica del Programa de Biología de la Universidad de Caldas (CEBUC), registro Humboldt No. 178.

Determinación de la calidad del agua. La calidad del agua de cada punto de muestreo se derterminó con los índices BMWP/Col (Biologycal Monitoring Working Party) adaptado para Colombia por Roldán (2003) y Zuñiga (2010) e implementado en el departamento de Caldas por Hahn-Von Hessberg, et al. (2009), Walteros-Rodríguez \& Daza (2009), Walteros-Rodríguez \& Paiba-Alzate (2010), González, et al. (2012) y Mesa-S, et al. (2013). El índice se contruye asignando valores de 1 a 10 a las familias de macroinvertebrados recolectados en campo. Los valores bajos $(1,2,3)$ son indicadores de aguas contaminadas y los valores altos (9 y 10), de aguas en perfecto estado. Los valores obtenidos indican el grado de tolerancia o la sensibilidad de cada grupo de macroinvertebrados a los contaminantes orgánicos y refleja la alteración del ecosistema en que habitan. Posteriormente, se calculó el índice ASPT (Average Score Per Taxon), es decir, el del puntaje promedio por taxon medido con valores de 1 a 10 , el cual se obtiene al dividir el valor del BMWP/Col entre el número de taxones incluidos en el cálculo. Los valores inferiores a 7 indican aguas con algún grado de contaminación. Por último, se determinó el índice EPT diseñado por Carrera \& Fierro (2001), el cual determina la abundancia de los macroinvertebrados de los órdenes Ephemeroptera, Plecoptera y Trichoptera (grupos sensibles a la contaminación), dividiéndola por la abundancia total. Los valores superiores a $75 \%$ indican aguas de buena calidad. Este índice ha sido empleado por Mesa-S, et al. (2013) en la cuenca del río Chinchiná-Caldas.

Analisis de datos. Se estimaron la riqueza y la abundancia de macroinvertebrados con base en el número de morfotipos recolectados en campo y se establecieron las diferencias entre los puntos de muestreo mediante un análisis de varianza de una via (ANOVA) con el programa estadístico IBM ${ }^{\circledR}$ SPSS ${ }$, versión 24 , previa confirmación de los supuestos de normalidad y homocedasticidad. Asimismo, se hizo un análisis de agrupación por dendrograma de similitud con el programa Past 2.17, y se calcularon los índices BMWP/Col, ASPT y EPT para determinar la calidad del agua en los puntos de muestreo.

\section{Resultados}

Diversidad de macroinvertebrados recolectados en campo. Se recolectaron 2.175 individuos agrupados en 15 órdenes, 58 familias y 97 morfotipos (Tabla 1). Los órdenes más abundantes fueron Trichoptera, Ephemeroptera y Coleoptera con el 39,26\%, 18,25\% y 17,23\% de la abundancia absoluta, respectivamente.

Entre los Ephemeroptera se presentaron 3 familias (Baetidae, Leptohyphidae, Leptophlebiidae) abarcando 13 géneros, siendo los mas abundantes Leptohyphes, Thraulodes y Baetodes. Los tricopteros representan 9 familias y 11 géneros, siendo Smicridea, Nectopsyche y Grumichella los mas abudantes, mientras que los elmidos fueron el grupo más abundante de los coleópteros.

A nivel de familia, Hydropsychidae, Leptoceridae y Elmidae $(13,47 \%, 12,64 \%$ y $11,86 \%$, respectivamente) dominaron en todos los puntos de muestreo, además de ser las familias que más morfotipos aportaron (Figura 2)

Al realizar los análisis estadisticos no se encontraron diferencias significativas en la riqueza $(p=0,09)$ ni en la abundancia $(p=0,78)$ entre los sitios evaluados (Figura 3 ). Sin embargo, se observó una mayor similitud entre los sitios de Sevilla y Cundinamarca (Figura 4).

Calidad del agua. La calidad del agua de los puntos de muestreo según el índice BMWP/Col (Roldán, 1999; Zúñiga, 2010) reportó valores superiores a 150, lo que indica aguas muy limpias, de buena calidad o no alteradas de manera evidente. Por su parte, el índice EPT (Carrera \& Fierro, 2001) indicó una muy buena calidad del agua en 
Tabla 1. Macroinvertebrados acuáticos recolectados en cada sitio de muestreo. Lac: vereda La Cruz, Sev: vereda Sevilla y Cun: vereda Cundinamarca

\begin{tabular}{|c|c|c|c|c|c|c|}
\hline \multirow[t]{2}{*}{ Orden } & \multirow[t]{2}{*}{ Familia } & \multirow[t]{2}{*}{ Géneros } & \multicolumn{3}{|c|}{ Estación } & \multirow[t]{2}{*}{ Total } \\
\hline & & & Lac & Sev & Cun & \\
\hline Gordoidea & Chordodidae & Chordodidae $1 *$ & 5 & 0 & 0 & 5 \\
\hline Isopoda & Philoscidae & Philoscidae $1 *$ & 6 & 1 & 2 & 9 \\
\hline Tricladidae & Planariidae & Dugesia & 2 & 1 & 19 & 22 \\
\hline Blattodea & Blattidae & Blattidae $1 *$ & 1 & 2 & 1 & 4 \\
\hline \multirow{30}{*}{ Coleoptera } & Cantharidae & Cantharidae $1 *$ & 0 & 1 & 0 & 1 \\
\hline & Chrysomelidae & Chrysomelidae 1 * & 0 & 1 & 2 & 3 \\
\hline & Curculionidae & Curculionidae $1 *$ & 0 & 1 & 1 & 2 \\
\hline & Dryopidae & Pelonomus & 7 & 0 & 6 & 13 \\
\hline & \multirow{3}{*}{ Dysticidae } & Dysticidae 1 & 3 & 0 & 0 & 3 \\
\hline & & Dysticidae 2 & 15 & 0 & 7 & 22 \\
\hline & & Dysticidae 3 & 1 & 0 & 3 & 4 \\
\hline & \multirow{12}{*}{ Elmidae } & Cylloepus & 7 & 16 & 20 & 43 \\
\hline & & Disersus & 0 & 4 & 2 & 6 \\
\hline & & Heterelmis & 23 & 19 & 26 & 68 \\
\hline & & Huleechius & 1 & 0 & 1 & 2 \\
\hline & & Macrelmis & 9 & 22 & 29 & 60 \\
\hline & & Microcylloepus & 12 & 8 & 9 & 29 \\
\hline & & Neoelmis & 1 & 4 & 1 & 6 \\
\hline & & Onychelmis & 1 & 0 & 1 & 3 \\
\hline & & Phanocerus & 9 & 3 & 6 & 18 \\
\hline & & Pharceonus & 9 & 1 & 1 & 11 \\
\hline & & Stegoelmis & 7 & 1 & 4 & 12 \\
\hline & & Xenelmis & 0 & 0 & 1 & 1 \\
\hline & Erotylidae & Erotylidae $1 *$ & 0 & 1 & 0 & 1 \\
\hline & Gyrinidae & Enhydrus & 3 & 0 & 1 & 4 \\
\hline & Heteroceridae & Heteroceridae 1 & 0 & 0 & 1 & 1 \\
\hline & Hydraenidae & Hydraenidae 1 & 1 & 0 & 0 & 1 \\
\hline & Lampyridae & Lampyridae $1 *$ & 0 & 1 & 1 & 2 \\
\hline & Psephenidae & Psephenops & 2 & 5 & 5 & 12 \\
\hline & \multirow{2}{*}{ Ptylodactilidae } & Anchytarsus & 6 & 8 & 9 & 23 \\
\hline & & Tetraglosa & 1 & 0 & 0 & 1 \\
\hline & Scirtidae & Scirtes & 2 & 0 & 1 & 3 \\
\hline & Staphylinidae & Stenus & 9 & 3 & 8 & 20 \\
\hline & Sydmaenidae & Scydmaenidae $1 *$ & 1 & 0 & 0 & 1 \\
\hline Dermaptera & labiidae & Labiidae $1 *$ & 0 & 1 & 1 & 2 \\
\hline \multirow{12}{*}{ Diptera } & Blephariceridae & Limonicola & 5 & 0 & 0 & 5 \\
\hline & Ceratopogonidae & Atrichopogon & 0 & 0 & 2 & 2 \\
\hline & \multirow{7}{*}{ Chironomidae } & Chironominae 1 & 1 & 1 & 3 & 5 \\
\hline & & Corynoneura & 0 & 0 & 5 & 5 \\
\hline & & Crycotopus & 9 & 5 & 18 & 32 \\
\hline & & Hudsonomyia & 1 & 0 & 0 & 1 \\
\hline & & Orthocladinae 1 & 2 & 0 & 0 & 2 \\
\hline & & Parochlus & 1 & 2 & 4 & 7 \\
\hline & & Stenochironomus & 5 & 0 & 0 & 5 \\
\hline & \multirow{2}{*}{ Culicidae } & Anopheles & 0 & 0 & 5 & 5 \\
\hline & & Culex & 0 & 0 & 6 & 6 \\
\hline & Dixidae & Dixella & 1 & 0 & 0 & 1 \\
\hline
\end{tabular}




\begin{tabular}{|c|c|c|c|c|c|c|}
\hline \multirow{5}{*}{ Diptera } & Simuliidae & Simulium & 40 & 8 & 11 & 59 \\
\hline & \multirow{4}{*}{ Tipulidae } & Hexatoma & 0 & 1 & 0 & 1 \\
\hline & & Limonia & 0 & 0 & 7 & 7 \\
\hline & & Molophilus & 0 & 1 & 1 & 2 \\
\hline & & Tipula & 1 & 0 & 1 & 2 \\
\hline \multirow{13}{*}{ Ephemeroptera } & \multirow{7}{*}{ Baetidae } & Andesiops & 0 & 0 & 3 & 3 \\
\hline & & Apobaetis & 0 & 0 & 1 & 1 \\
\hline & & Baetodes & 17 & 24 & 19 & 60 \\
\hline & & Camelobaetidius & 0 & 0 & 2 & 2 \\
\hline & & Mayobaetis & 13 & 7 & 14 & 34 \\
\hline & & Nanomis & 10 & 7 & 15 & 32 \\
\hline & & Prebaetodes & 1 & 4 & 0 & 5 \\
\hline & \multirow{4}{*}{ Leptohyphidae } & Leptohyphes & 48 & 23 & 70 & 141 \\
\hline & & Traveryphes & 0 & 0 & 1 & 1 \\
\hline & & Farrodes & 3 & 4 & 0 & 7 \\
\hline & & Terpides & 0 & 0 & 1 & 1 \\
\hline & \multirow{2}{*}{ Leptophlebiidae } & Thraulodes & 20 & 61 & 28 & 109 \\
\hline & & Ulmeritoides & 0 & 0 & 1 & 1 \\
\hline \multirow{9}{*}{ Hemiptera } & Cercopidae & Cercopidae $1 *$ & 0 & 1 & 0 & 1 \\
\hline & & Cicadellidae $1^{*}$ & 1 & 0 & 0 & 1 \\
\hline & Cicadellidae & Cicadellidae $2 *$ & 1 & 0 & 0 & 1 \\
\hline & Gelastocoridae & Gelastocoridae & 1 & 1 & 0 & 1 \\
\hline & Hebridae & Hebrus & 1 & 1 & 1 & 3 \\
\hline & Mesovelidae & Mesovelia & 2 & 0 & 0 & 2 \\
\hline & Naucoridae & Cryphocricos & 4 & 0 & 3 & 7 \\
\hline & \multirow{2}{*}{ Veliidae } & Limnocoris & 33 & 12 & 12 & 57 \\
\hline & & Rhagovelia & 8 & 0 & 3 & 11 \\
\hline Lepidoptera & Crambidae & Petrophila & 2 & 4 & 5 & 11 \\
\hline Megaloptera & Corydalidae & Corydalus & 0 & 4 & 7 & 11 \\
\hline \multirow{6}{*}{ Odonata } & Aeshnidae & Aeshnidae 1 & 0 & 1 & 1 & 2 \\
\hline & Gomphidae & Gomphidae 1 & 1 & 0 & 0 & 1 \\
\hline & Lestidae & Archilestes & 2 & 0 & 1 & 3 \\
\hline & Libellulidae & Libellula & 1 & 0 & 0 & 1 \\
\hline & Megapodagrionidae & Sciotropis & 0 & 0 & 1 & 1 \\
\hline & Polythoridae & Polythore & 22 & 0 & 3 & 25 \\
\hline Plecoptera & Perlidae & Anacroneuria & 30 & 63 & 121 & 214 \\
\hline Socodea & Socodea 1 & Socodea $1 *$ & 4 & 0 & 0 & 4 \\
\hline \multirow{11}{*}{ Trichoptera } & Calamoceratidae & Phylloicus & 36 & 1 & 6 & 43 \\
\hline & Glossosomatidae & Culoptila & 0 & 2 & 1 & 3 \\
\hline & Helicopsychidae & Helicopsyche & 20 & 53 & 51 & 124 \\
\hline & \multirow{2}{*}{ Hydropsychidae } & Leptonema & 45 & 13 & 28 & 86 \\
\hline & & Smicridea & 102 & 43 & 62 & 207 \\
\hline & Hydroptilidae & Metrichia & 0 & 0 & 1 & 1 \\
\hline & \multirow{2}{*}{ Leptoceridae } & Grumichella & 17 & 93 & 33 & 143 \\
\hline & & Nectopsyche & 21 & 49 & 62 & 132 \\
\hline & Odontoceridae & Marilia & 1 & 0 & 3 & 4 \\
\hline & Philopotamidae & Chimarra & 17 & 53 & 22 & 92 \\
\hline & Polycentropidae & Polycentropus & 5 & 10 & 5 & 20 \\
\hline \multicolumn{3}{|l|}{ Total } & 698 & 658 & 819 & 2175 \\
\hline
\end{tabular}

*:taxones con hábitos terrestres 
todos los puntos de muestreo, en tanto que la puntuación en el índice ASPT indicó la presencia de aguas con algún grado de contaminación, siendo más evidente en la vereda Sevilla (Tabla 2).

\section{Discusión}

Diversidad de macroinvertebrados recolectados en campo. Al igual que en este estudio, en varias regiones del país se ha reportado una gran diversidad de grupos de macroinvertebrados indicadores de la buena calidad del agua (efemerópteros, plecópteros y tricopteros). Por ejemplo, Zúñiga, et al. (2013) y Giraldo, et al. (2014) lo han hecho en pequeñas quebradas del Valle del Cauca; Tamaris-T, $\boldsymbol{e t}$ al. (2007) en el río Gaira-Sierra Nevada de Santa Marta, Árias-Díaz, et al. (2007) en la cuenca del río Coello (Tolima), Gutiérrez \& Días (2015) en 55 fuentes hídricas de 12 municipios en el departamento de Caldas y Gonzáles, et al. (2012) en la cuenca del río Chinchina.

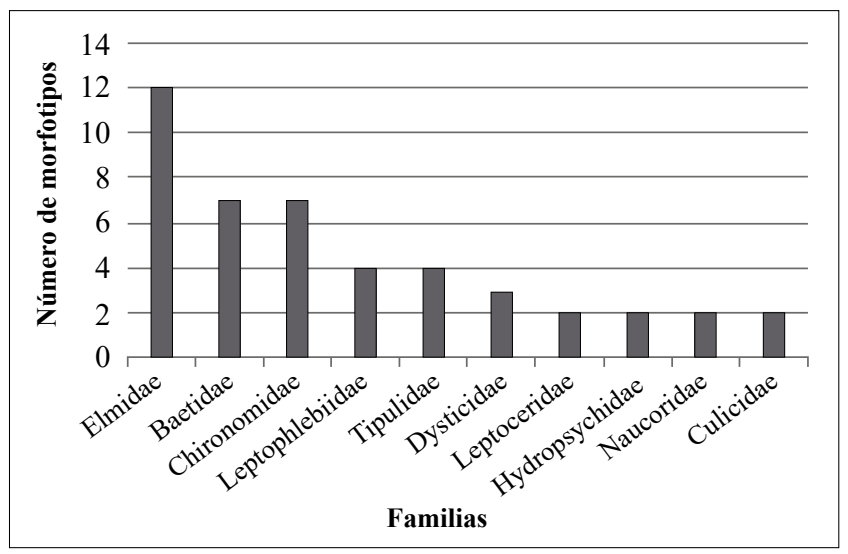

Figura 2. Relación entre el número de morfotipos y familias de los individuos recolectados en los tres sitios de muestreo de la quebrada Santo Tomás-Pensilvania
El orden Ephemeroptera fue uno de los mas abundantes y diversos y agrupando a $50 \%$ de las familias y los géneros descritos para el departamento de Caldas por Gutierrez \& Días (2015), quienes observaron seis familias y 26 géneros en su estudio.

La gran diversidad de este grupo es un aspecto clave para futuros proyectos de biomonitorización en la zona de estudio, teniendo en cuenta que se consideran sensibles a la contaminación e indicadores de la presencia de aguas en buen estado de conservación (Roldán, 1999; Zúñiga, 2010), aspecto clave para esta pequeña quebrada que abastece varios acueductos rurales.

Los tricópteros también son comunes en la cuenca de la quebrada Santo Tomás y comprendieron nueve familias y 11 géneros, siendo Hydropsychidae y Leptoceridae los mas abundantes. La diversidad de este grupo es importante en la zona de estudio, aunque fue menor a la reportada en otras regiones del Neotrópico como Costa Rica (Springer, 2010), en donde se conocen 15 familias y 52 géneros. En Colombia, en una revisíon de Muñoz-Quesada (2010) se han descrito 45 géneros y 210 especies, y quizás el departamento mejor explorado es Antioquia, donde Flint (1991) describió 124 especies en 37 géneros y 14 familias, en tanto que Posada \& Roldán (2003) encontraron 13 familias y 35 géneros en 84 ecosistemas lóticos y lénticos de todo el departamento. En el Tolima, Guevara, et al. (2005) observaron 12 familias y 26 taxones en 29 estaciones del río Coello y Vásquez-Ramos, et al. (2007), 11 familias y 26 géneros en 27 estaciones de muestreo en el río Totare.

En Caldas, Hahn-Von Hessberg, et al. (2009) reportaron la presencia de Trichoptera, con cinco familias en el municipio de Palestina. Por su parte, Walteros-Rodríguez \& Paiba-Alzate (2009) lo registraron en las quebradas El Diamante, La Mula y en el río Chinchina (Manizales) con ocho familias, y Gonzalez, et al. (2012) encontraron 11 familias y 20 géneros en la cuenca del río Chinchina, en tanto
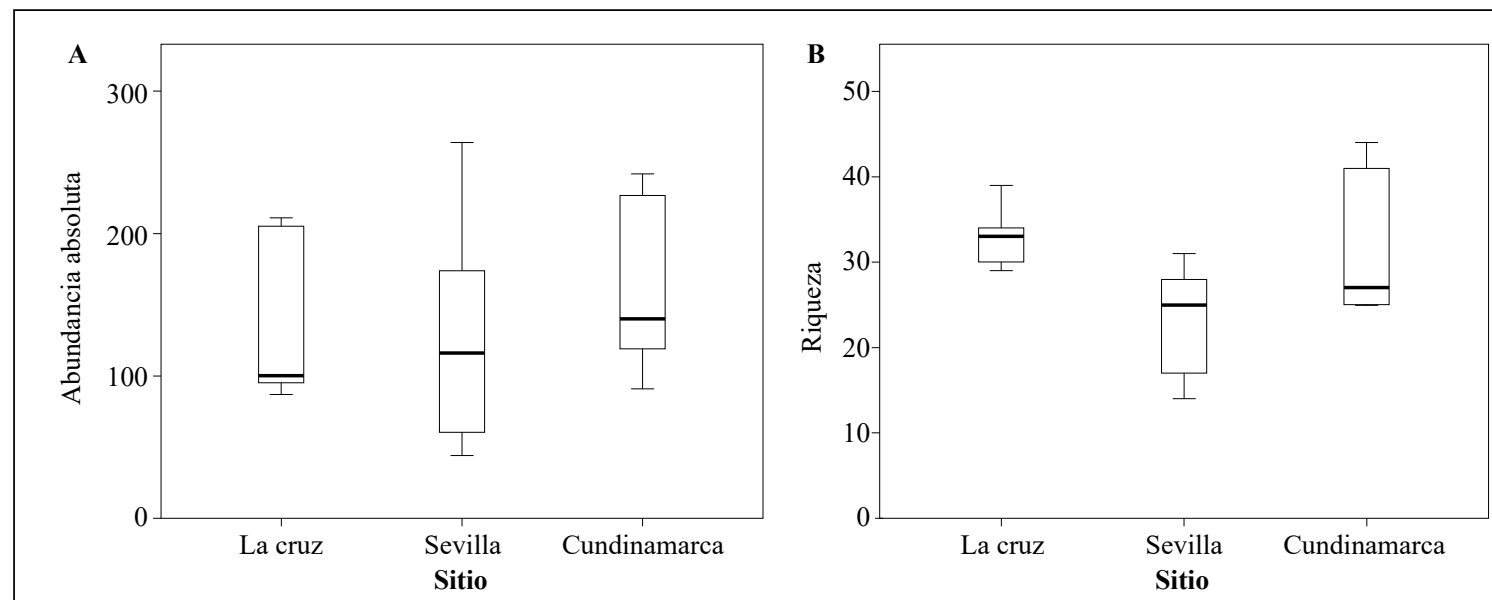

Figura 3. Diagrama de cajas y bigotes de la comparación de la abundancia absoluta (A) y la riqueza (B) en los sitios de muestreo de la quebrada Santo Tomás 


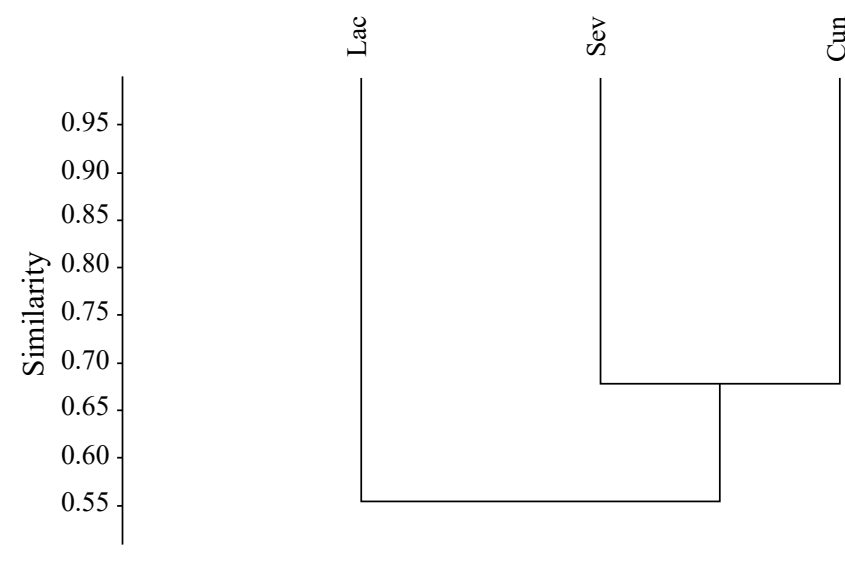

Figura 4. Dendrograma de similitud basado en la distancia de Bray-Curtis con el método de enlace UPGMA para los sitios de muestreo (Lac: vereda La Cruz, Sev: vereda Sevilla y Cun: vereda Cundinamarca) en la quebrada Santo Tomás-Pensilvania

Tabla 2. Calidad del agua en los diferentes sitios de muestreo en la quebrada Santo Tomás-Pensilvania

\begin{tabular}{lccc}
\hline $\begin{array}{l}\text { Índice/sitio } \\
\text { de muestreo }\end{array}$ & $\begin{array}{c}\text { Vereda } \\
\text { La Cruz }\end{array}$ & $\begin{array}{c}\text { Vereda } \\
\text { Sevilla }\end{array}$ & $\begin{array}{c}\text { Vereda } \\
\text { Cundinamarca }\end{array}$ \\
\hline BMWP/Col & 194 & 167 & 207 \\
ASPT & 6,2 & 3,8 & 5,8 \\
EPT & 87 & 85 & 93 \\
\hline
\end{tabular}

que Mesa-S, et al. (2012), siete familias y 12 géneros en una subcuenca del río Chinchiná (Manizales). No obstante, los reportes para el oriente del departamento son escasos, y solamente Walteros-Rodríguez \& Daza-Castro (2009) han publicado registros para el municipio de Manzares, ubicado a $75 \mathrm{~km}$ del lugar de muestreo del presente estudio.

Este grupo fue mas abundante en el primer sitio de muestreo (vereda La Cruz), lugar donde se encuentra una reserva administrada por la junta del acueducto local desde 1999, lo que puede estar relacionado con una mayor oferta de refugio y alimento en este sitio que en los otros, favoreciendo así la diversidad.

Dentro del orden Coleoptera la familia más diversificada en ecosistemas acuáticos son los Elmidae. Según Manzo (2013), los élmidos son abundantes en la región neotropical, con 46 géneros descritos actualmente. En el presente estudio se encontró una gran diversidad de este grupo, con 12 géneros, lo que corresponde al $26 \%$ de los registros para el Neotrópico. Los grupos más abundantes fueron los Heterelmis $(26,35 \%)$ y los Macrelmis $(23,26 \%)$, resultados similares a los registrado por Árias-Días, et al. (2007) para el río Coello (Tolima) y por Meza, et al. (2012) y Gonzáles, et al. (2013) en el río Chinchiná (Manizales). Para evitar errores en la determinación de este grupo, se revisó el estudio de Sondermann (2013) sobre su taxonomía y distribución en Colombia.
Contrario a lo que se esperaba, no se presentaron diferencias estadísticas en la riqueza $(\mathrm{p}=0,09)$ ni la abundancia $(p=0,78)$ de macroinvertebrados en los sitios de muestreo, aunque el análisis de conglomerados evidenció una mayor similitud entre las veredas de Sevilla y Cundinamarca (sitios 2 y 3). Según Giraldo, et al. (2014), las quebradas situadas en zonas agrícolas y ganaderas (Valle del Cauca) suelen presentar diferencias significativas comparadas con los sitios bien conservados. En el presente estudio tales diferencias podrían no haberse visto reflejadas debido a un marcado gradiente altitudinal reflejado en un recambio continuo de especies, tal como lo propone Vanotte (1980) en la teoría del río continuo. En este sentido, se encontraron diez morfotipos exclusivos de la zona conservada (La Cruz), seis del punto con descargas residuales (Sevilla) y 13 del punto con intervención agrícola y ganadera (Cundinamarca). En la vereda Sevilla los grupos exclusivos, como las familias Chironomidae, Culicidae y Tipulidae, son indicadores de aguas perturbadas, lo que reflejaría alguna alteración en el ecosistema, aunque no se vea reflejada en una disminución obstensible de la diversidad.

Calidad del agua según los índices BMWP/Col, EPT y ASPT. Según las puntuaciones de los los índices BMWP/ Col y EPT, las aguas de la quebrada Santo Tomás son de muy buena calidad. Estos resultados son similares a los encontrados por Giacometti \& Bersosa (2006) en el río Alambi (Ecuador), así como por González, et al. (2012) y Mesa-S, et al. (2012) en la cuenca del río Chinchiná (Caldas) y por Hahn-Von Hessberg, et al. (2009) en la estación piscícola de la Universidad de Caldas (municipio de Palestina). Otros autores como Walteros-Rodrígez y Paiba-Alzate (2010), en las quebredas El Diamante, La Mula y Río Chinchiná (Manizales), y Walteros-Rodríguez \& Daza-Castro (2009) en el municipio de Manzares en el río Guarinó, observaron variaciones en la calidad del agua de las zonas altas de la cuenca y de las bajas, siendo estas últimas las de más baja calidad.

El índice ASPT evidenció aguas moderadamente contaminadas. En Colombia, Arango, et al. (2008), en las quebradas La Cristalina y La Risaralda (San Luis-Antioquia), reportaron valores similares al encontrado en el presente estudio, en tanto que Ramírez, et al. (2013) observaron aguas muy contaminadas en el río Bogotá y Mosquera y Mosquera (2017) aguas ligeramente contaminadas en el río San Juan (Chocó). En cuanto al departamento de Caldas, no se encontraron reportes del uso de este índice.

\section{Conclusiones}

Los índices biológicos empleados en el presente estudio indicaron la presencia de aguas de buena calidad. Sin embargo, se recomienda evaluar la variación temporal e incluir análisis fisicoquímicos para indagar sobre el comportamiento de la riqueza y la abundancia de los macroinvertebrados, así como sobre el efecto de las actividades antrópicas en la zona de estudio. 


\section{Agradecimientos}

Al laboratorio de análisis fisicoquímicos del Centro Pecuario y Agroempresarial del SENA en la subregional de La Dorada, Caldas, y al laboratorio de ecología de la Universidad de Caldas, por permitirnos realizar la fase de identificación de los macroinvertebrados en sus instalaciones.

A la Colección Entomológica del Programa de Biología de la Universidad de Caldas (CEBUC), por recibir el material recolectado en este estudio.

A los lectores anónimos que ayudaron a fortalecer la redacción de este documento.

\section{Contribución de los autores}

Sergio Adrián Murillo: participó en la etapa de investigación. Todos los coinvestigadores participamos coactivamente en la investigación y procesamiento de la información colectada en el campo, parte del material fue identificado en el laboratorio de ecología de la Universidad de Caldas por Sergio Adrián Murillo, y otro tanto, fue identificado en el laboratorio de análisis fisicoquímico del Centro Pecuario y Agroempresarial de La Dorada, Caldas, por los investigadores: Edwin Santiago Restrepo Bastidas, Alexander Mendoza Mora y aprendiz Sena.

\section{Conflicto de intereses}

Los autores declaramos que no existen conflictos de intereses que pongan en riesgo la validez de los resultados presentados.

\section{Referencias}

Agroclima. (2015). Plataforma agrícola cafetera. Fecha de consulta: 1 de junio de 2018. Disponible en: https://agroclima. cenicafe.org/registros-historicos?p_auth=SQSxcwp\&p_ pid=datos_historicos_WAR_datos_historicosportlet\&p_p_ lifecycle $=1 \& p \_p \_s t a t e=$ normal\&p_p_mode $=$ view $\&$ p_p_ col_id=column-2\&p_p_col_count $=3 \&$ p_p_col_ pos $=2 \&$ \&atos_historicos_WAR_datos_historicosportlet facesViewId Render $=\% 2$ Fviews $\% 2$ Fview.xhtml

Arango, M.C., Álvarez, L.F., Arango, G.A., Torres, O.E., Monsalve, A.J. (2008). Calidad del agua de las quebradas La Cristalina y La Risaralda, San Luis Antioquia. Revista Escuela de Ingeniería de Antioquia. 9: 121-141.

Árias-Días, D.M., Reinoso-Flórez, G., Guevara-Cardona, G. (2007). Distribución espacial y temporal de los coleópteros acuáticos en la cuenca del rio coello (Tolima-Colombia). Caldasia. 29 (1): 177-194.

Carrera, C. Fierro, K. (2001). Manual de monitoreo: los macroinvertebrados acuáticos como indicadores de la calidad del agua. Ecociencia-Quito. p. 57.

Corpocaldas. (1999). Agenda para la gestión ambiental del municipio de Pensilvania. Corporación Autónoma Regional de Caldas, Manizales. p. 1-105.

Delong, M.D., Brusven, M.A. (1998). Macroinvertebrate community structure along the longitudinal gradient of an agriculturally impacted stream. Environmental Management. 22 (3): 445-457.

Domínguez, E. Fernández, H. (2009). Macroinvertebrados bentónicos sudamericanos, sistemática y taxonomía. Primera edición, Tucuman. Fundación Miguel Lillo. p. 1-652.
Dudgeon, D. Arthington, A.H., Gessner, M.O., Kawabata, Z., Knowler, D.J., Léveque, C., Naiman, R.J.. Prieur-Richard, A.H., Soto, D., Stiassny, M.I. Sullivan, C.A. (2006). Freshwater biodiversity: Importance, threats, status and conservation challenges. Biological Reviews. 81: 163-182.

Fernández, l. Springer, M. (2008). El efecto del beneficiado del café sobre los insectos acuáticos en tres ríos del valle central (alajuela) de Costa Rica. Revista de Biologia Tropical. 56 (Suppl. 4): 237-256.

Fierro, P., Bertran, C., Mercado, M., Peña-Cortés, F., Tapia, J., Hauensteind, E., Caputo. I., Vargas-Chacoff, I. (2015). Landscape composition as a determinant of diversity and functional feeding groups of aquatic macroinvertebrates in southern river of the Araucanía, Chile. Latin American Journal of Aquatic Research. 43 (1): 186-200.

Flint, O.S., Jr. (1991). Studies of neotropical caddisflies, XVL: The taxonomy, phenology and faunistics of the Trichoptera of Antioquia, Colombian. Smithsonian Contributions of Zoology. 520: 1-113.

Giacometti, J.C., Bersosa F. (2006). Macroinvertebrados acuáticos y su importancia como indicadores de calidad del agua en el río Alambi. Serie Zoológica. 2: 17-32.

Giraldo, J.P., Chará, J., Zuñiga, M.C., Chará-Serna, A.M. Pedraza, G. (2014). Impacto del uso del suelo agropecuario sobre los macroinvertebrados acuáticos en pequeñas quebradas del río la vieja (Valle del Cauca-Colombia). Revista de Biología Tropical. 62 (Suppl. 2): 203-219.

González, S.M., Ramírez, Y.P., Meza, A.M. Días, L.G. (2012). Diversidad de macroinvertebrados acuáticos y calidad de agua de quebradas abastecedoras del municipio de Manizales. Boletín Científico del Centro de Museos de Historia Natural, Universidad de Caldas. 16 (2): 135-148.

Guevara-Cardona, G., Reinoso-Flórez, G. Villa, F. (2005). Estudio del orden Trichoptera en su estado larval en la cuenca del río Coello, departamento del Tolima. Revista Asociación Colombina de Ciencias Biológicas. 17: 59-70.

Gutiérrez, Y., Días, L.G. (2015). Ephemeroptera (insecta) de Caldas-Colombia, claves taxonómicas para los géneros y notas sobre su distribución. Papéis Avulsos de Zoología. 55 (2): 13-46.

Hahn-Von Hessberg, C.M, Toro, D.R, Grajales-Quintero, A, Duque-Quintero, G.M, Serna-Uribe, L. (2009) Determinación de la calidad del agua mediante indicadores biológicos y fisicoquímicos en la estación piscícola, Universidad de Caldas, municipio de Palestina, Colombia. Boletín Científico Centro de Museos, Museo de Historia Natural. 13 (2): 89-105.

Heckman, C. (2006). Encyclopedia of South American aquatic insects: Odonata- Anisoptera. Illustrated keys to known families, genera and species in South América. Dordrecht, The Netherlands. Springer Eds. 1-731.

Heckman, C. (2011). Encyclopedia of South American aquatic insects: Odonata- Zygoptera. Illustrated keys to known families, genera and species in South América. Olympia Washington, USA. Springer Eds. 1-694.

Jonsson, M., Malmqvist, B. Hoffsten, P.E. (2001). Leaf litter breakdown rates in boreal streams: Does shredder species richness matter? Freshwater Biology. 46: 161-171.

Jorcin, A. Nogueira, M.G. (2008). Benthic macroinvertebrates in the Paranapanema reservoir cascade (southeast Brazil). Brazilian Journal of Biology. 68 (4): 1013-1024. 
Manzo, V. (2005). Key to the South American genera of Elmidae (Insecta-Coleoptera) with distributional date. Studies of Neotropical Fauna and Environment. 40 (3): 201-208.

Manzo, V. (2013). Los élmidos de la región neotropical (ColeopteraByrrhoidea: Elmidae), diversidad y distribución. Revista de la Sociedad Entomológica Argentina. 72 (3-4): 199-212.

Meza, A.M., Rubio-M, J., Días, L.G. Valteros, J.M. (2012). Calidad de agua y composición de macroinvertebrados acuáticos en la subcuenca alta del río Chinchiná. Caldasia. 34 (2): 443-456.

Mosquera, Z. Mosquera, M.M. (2017). Diversidad de la entomofauna acuática y calidad de agua de quebradas del río San Juan, Chocó-Colombia. Revista Univeridad de Ciencias Aplicadas y Ambientales, Actualidad y Diversidad Científica. 20 (1): 149-161.

Muñoz-Quesada, F. (2000). Especies del orden Trichoptera (Insecta) en Colombia. Biota Colombiana. 1 (3):267-288.

Posada, J.A. Roldán, G. (2003). Clave ilustrada y diversidad de larvas de Trichoptera en el nor-occidente de Colombia. Caldasia. 25 (1): 169-192.

Ramírez, A. (2010). Capítulo 2, Métodos de recolección. Revista de Biología Tropical (en línea). Fecha de consulta: 22 de febrero de 2018. Disponible en: http://www.redalyc.org/ articulo.ao?id=44922967002

Ramírez, D.F., Talero, G.M., López, R.H. (2013). Macroinvertebrados bentónicos y calidad del agua en un tramo del río Bogotá, Cajicá-Colombia. Revista Univeridad de Ciencias Aplicadas y Ambientales, Actualidad y Diversidad Científica. 16 (1): 205-2014.

Roldán, G. (1999). Los macroinvertebrados y su valor como indicadores de la calidad del agua. Revista de la Academia Colombiana de Ciencias Exactas, Físicas y Naturales. 23 (88): 175-187.

Sondermann, W. (2013). It's the elmids of colombia strongly marked by neartics elements? A remote analysis of genera names provided in 30 recently published benthic macroinvertebrate assessment: Coleoptera: Byrrhoidea: Elmidae. Dugesia. 20 (2): 251-260.
Springer, M. (2010). Capítulo 7, Trichoptera. San José-Costa Rica. Revista de Biología Tropical. 58 (Suppl. 4): 151-198.

Sweeney, B.W., Bott, T.I., Jackson, J.K., Kaplan, I.A., Newbold, J.D., Standley, I.J. Horwitz, R.J. (2004). Riparian deforestation, stream narrowing, and loss of stream ecosystem services. Proceedings of the National Academy of Sciences of the United States of America. 101 (39): 14132-14137.

Tamaris-T, C.E., Turizo-C, R.R., Zuñiga, M.C. (2007). Distribución espacio-temporal y hábitos alimenticios de ninfas de Anacroneuria (Insecta: Plecoptera: Perlidae) en el río Gaira (Sierra Nevada de Santa Marta, Colombia). Caldasia. 29 (2): 375-385.

Vannote R.L., Minshall, G.W., Cummins, K.W., Sedell, J.R. Cushing, C.C. (1980). The river continuum concept. Canadian Journal of Fisheries and Aquatic Science. 37: 130-137.

Vásquez-Ramos, J.M., Ramírez-Días, F., Reinoso-Flórez, G., Guevara-Cardona, G. (2010). Distribución espacial y temporal de los tricópteros inmaduros en la cuenca del río Totare (Tolima-Colombia). Caldasia. 32 (1): 129-148.

Walteros-Rodríguez, J.M., Daza-Castro, J.F. (2009). Caracterización hidrobiológica y evaluación ecológica de la calidad del agua del rio Guarinó, departamento de Caldas. Revista de Ciencias. 12: 31-44.

Walteros-Rodríguez, J.M., Paiba-Alzate, J.E. (2010). Estudio preliminar de la comunidad de macroinvertebrados acuáticos en la reserva forestal Torre Cuatro. Boletín Científico Centro de Museos, Museo de Historia Natural. 14 (1): 137-149.

Zuñiga, M.C. (2010). Capitulo 7, Bioindicadores de calidad de agua y caudal ambiental. En: J.R.K. Cantera y E. Carvajal \& L.M.H. Castro (editores). El caudal ambiental: conceptos, experiencias y desafíos. p. 167-197. Programa Editorial Universidad del Valle, Cali-Colombia.

Zuñiga, M.C., Chará, J., Giraldo, I.P., Chara-Serna, A.M. Pedraza, G.X. (2013). Composición de la comunidad de macroinvertebrados acuáticos en pequeñas quebradas de la región andina colombiana, con énfasis en la entomofauna. Dugesia. 20 (2): 263-277. 